\title{
Role of thermal strain in the metal-insulator and structural phase transition of epitaxial $\mathrm{VO}_{2}$ films
}

\author{
V. Théry, ${ }^{1}$ A. Boulle, ${ }^{1}$ A. Crunteanu,${ }^{2}$ J. C. Orlianges,${ }^{1}$ A. Beaumont,${ }^{2}$ \\ R. Mayet, ${ }^{1}$ A. Mennai, ${ }^{2}$ F. Cosset,${ }^{2}$ A. Bessaudou, ${ }^{2}$ and M. Fabert ${ }^{2}$ \\ ${ }^{1}$ Sciences des Procédés Céramiques et Traitements de Surfaces, CNRS UMR 7315, \\ Centre Européen de la céramique, 12 rue Atlantis, 87068 Limoges Cedex, France \\ ${ }^{2}$ XLIM, UMR 7252 CNRS/ University of Limoges, \\ 123 Av. Albert Thomas, 87060 Limoges, France.
}

\begin{abstract}
The metal-insulator switching characteristics of $\mathrm{VO}_{2}$ play a crucial role in the performances of $\mathrm{VO}_{2}$-based devices. In this work we study high-quality (010)-oriented epitaxial films grown on (001) sapphire substrates by means of electron-beam evaporation and investigate the role of interface defects and thermal strain on the parallel evolution of the metal-insulator transition (MIT) and structural phase transition (SPT) between the monoclinic (insulator) and rutile (metal) phases. It is demonstrated that the highly-mismatched $\mathrm{VO}_{2} / \mathrm{Al}_{2} \mathrm{O}_{3}$ interface promotes a domain-matching epitaxial growth process where the film grows in a strain-relaxed state and the lattice distortions are confined at the interface in regions with limited spatial extent. Upon cooling down from the growth temperature, tensile strain is stored in the films as a consequence of the thermal expansion mismatch between $\mathrm{VO}_{2}$ and $\mathrm{Al}_{2} \mathrm{O}_{3}$. The thinnest film exhibit the highest level of tensile strain in the interfacial plane resulting in a shift of both the MIT and the SPT temperatures towards higher values, pointing to a stabilization of the monoclinic / insulating phase. Concomitantly, the electrical switching characteristics are altered (lower resistivity ratio and broader transition) as result of the presence of structural defects located at the interface. The SPT exhibits a similar evolution with, additionally, a broader hysteresis due to the formation of an intermediate, strain-stabilized, phase in the M1-R transition. Films with thickness ranging between 100-300 nm undergo a partial strain relaxation and exhibit the best performances, with a sharp $\left(10^{\circ} \mathrm{C}\right.$ temperature range $)$ and narrow (hysteresis $<4^{\circ} \mathrm{C}$ ) MIT extending over more than four orders of magnitude in resistivity $\left(6 \times 10^{4}\right)$.
\end{abstract}

\section{INTRODUCTION}

Vanadium dioxide $\left(\mathrm{VO}_{2}\right)$ is considered as an archetypal strongly electron correlated material undergoing a reversible first order metal-to-insulator transition (MIT) at a temperature $T_{M I T}$ around $68^{\circ} \mathrm{C}^{1}$. It exhibits a high-resistivity insulating phase for $T<T_{M I T}$ and a metallic behavior for $T>T_{M I T}$, with up to five orders of magnitude change in electrical resistivity between the two states and intrinsic transition timescales down to $100 \mathrm{fs}^{2}$. These properties triggered an increased interest for its possible integration in electronic and optical devices, such as high-speed switches and Mott-field effect transistors ${ }^{3-6}$, microwave switch devices ${ }^{7,8}$, or optical detectors/ switches ${ }^{9,10}$. In these applications, the switching characteristics of $\mathrm{VO}_{2}$ (resistivity ratio, switching hysteresis, etc.) play a crucial role in the device performances ${ }^{6,11}$.

The MIT in $\mathrm{VO}_{2}$ is accompanied by a structural phase transition (SPT) from a low-temperature insulating monoclinic M1 phase to a high temperature rutile $\mathrm{R}$ phase. Although the simultaneity and the causal relationship between the MIT and the SPT, as well as the possible role of intermediate phases occurring during the transition are still the subject of intense research, it is now well established that structural defects ${ }^{12-15}$ and strain are major factors affecting the switching characteristics. In particular, the role of strain in the MIT of $\mathrm{VO}_{2}$ materials has been thoroughly studied in the last decades, both from a theoretical ${ }^{16-18}$ and experimental ${ }^{19-26}$ point of view. Regarding thin film systems, the most interesting conclusions could be drawn from $\mathrm{VO}_{2}$ film epitaxially grown on
$\mathrm{TiO}_{2}$ substrates with various orientations ${ }^{20,21,27-29}$. In brief, from these studies it appears that the SPT and the MIT are decoupled ${ }^{30,31}$ and that uniaxial tensile strain exerted along the $\mathrm{c}$ axis of the $\mathrm{R}$ phase (i.e. along the direction of the formation of the $\mathrm{V}-\mathrm{V}$ dimers) opens the gap in the insulating phase and shifts the MIT towards higher temperatures. In the case of $\mathrm{VO}_{2}$ films grown on (001) sapphire substrates, the situation is less clear. Whereas, a shift of the MIT temperature under the action of strain is sometimes observed ${ }^{32-34}$, other reports do not indicate such a shift but rather a degradation of the switching characteristics ${ }^{14,35}$. Moreover, in these studies, little or no consideration is given to the SPT.

In this work we investigate the role of strain of the on both the MIT and the SPT in epitaxial $\mathrm{VO}_{2}$ films grown on (001) sapphire substrates by electron beam evaporation. We demonstrate that, because of the large latticematch between $\mathrm{VO}_{2}$ and $\mathrm{Al}_{2} \mathrm{O}_{3}$, the films grow in a strain-relaxed state and that tensile strain is stored in the films upon cooling down from growth temperature as a result of the thermal expansion mismatch between the film and the substrate. Tensile strain produces a shift of both the SPT and the MIT towards higher temperatures, whereas the presence of structural defects located at the interface alter the switching characteristics. Finally we discuss the role of thermal strain in the stabilization of an intermediate phase between the M1 and the R phases. 


\section{EXPERIMENTAL}

\section{A. Film growth}

The $\mathrm{VO}_{2}$ layers were obtained inside a high-vacuum chamber (evacuated to a base pressure of $5 \times 10^{-5} \mathrm{~Pa}$ prior to the deposition) using the electron-beam evaporation of a metallic vanadium target under oxygen atmosphere (working pressure $\left.\sim 8 \times 10^{-2} \mathrm{~Pa}\right)^{36}$. Typically, thin films having thicknesses between 15 and $600 \mathrm{~nm}$ were grown at deposition rates not exceeding $0.05 \mathrm{~nm} . \mathrm{s}^{-1}$ on c-cut (001) sapphire substrates heated at $500^{\circ} \mathrm{C}$. After the growth process, the films were cooled down to room temperature and were submitted to a post-deposition annealing process at $550^{\circ} \mathrm{C}$ under oxygen atmosphere (base pressure of $0.5 \mathrm{~Pa}$ ) for 15 minutes. The obtained films are highly uniform, with homogeneous thickness and properties (structural, electrical, optical) even on sapphire wafers with diameters as large as two-inches, with rootmean squared roughness of $2.4 \mathrm{~nm}$ as recorded by atomic force microscopy. All the $\mathrm{VO}_{2}$ films presented further on this paper were obtained on similar $20 \times 20 \mathrm{~mm}^{2}$ c-cut sapphire substrates.

\section{B. Electrical properties}

The electrical resistivity variation with temperature across the MIT of the $\mathrm{VO}_{2}$ films was recorded in the $25-95^{\circ} \mathrm{C}$ temperature interval and have been measured using the four-probes technique. We used a custom setup employing four in-line spring-loaded probes (spaced by $1 \mathrm{~mm}$ ) coupled to a Keithley 2612B sourcemeter. A Peltier element was used to control the heating/cooling of the samples while the temperature was monitored by a Pt-100 thermocouple attached near the films' surfaces.

\section{Structural properties}

Raman spectroscopy measurements have been carried out using a Raman inverted microscope (Horiba LabRAM HR Evolution) using a continuous HeNe laser light $(\lambda=632.8 \mathrm{~nm})$ with an incident power of $6 \mathrm{~mW}$ and focused on the sample with a $60 \times$ objective (Nikon S plan fluor ELWD, numerical aperture of 0.7 ) to a spot of $\sim 2$ $\mu \mathrm{m}$ in diameter. Temperature-dependent measurements were performed using the same Peltier element used for the electrical characterization of the samples.

$\mathrm{X}$-ray diffraction experiments (XRD) have been performed on a Bruker "D8 discover" diffractometer equipped with a parabolic multilayer mirror, a tworeflection asymmetrically cut $\mathrm{Ge}(220)$ monochromator ( $\mathrm{Cu}$ Ka1 radiation) as primary optics and linear position sensitive detector covering a $2^{\circ} 2 \theta$ range with a $0.01^{\circ}$ angular resolution. $\theta-2 \theta$ scans performed over a wide $2 \theta$ range $\left(2 \theta=10-110^{\circ}\right)$ revealed that all films correspond to the $\mathrm{VO}_{2}$ phase with a single (010) orientation with respect to the underlying (001) sapphire substrate, see
Fig. 1(a). Moreover, grazing incidence diffraction (scanning $2 \theta$ at a fixed $\omega$ incidence) evidenced the absence of any disoriented phase (data not shown here). The inplane orientation of the films have been determined by performing XRD $\phi$-scans, using the (220) reflection of $\mathrm{VO}_{2}$ and the (104) reflection of sapphire, Fig. 1(c).

High-resolution $\theta-2 \theta$ scans and $\omega$-scans (scanning incidence angle $\omega$ at fixed $2 \theta$ ) have been performed through the (020) and (040) reflections of $\mathrm{VO}_{2}$. The former scans give access to structural information in the direction normal to the surface (out-of-plane direction), such as the film thickness and the level of strain. The latter $\omega$-scans provide structural information in the direction parallel to the surface (in-plane direction), such as the mosaicity and the lateral coherence length. Temperature-dependent XRD experiments, using a Peltier heating element, with temperature varying between $50^{\circ} \mathrm{C}$ and $90^{\circ} \mathrm{C}$ have been performed in order to monitor the structural phase transition in the films.
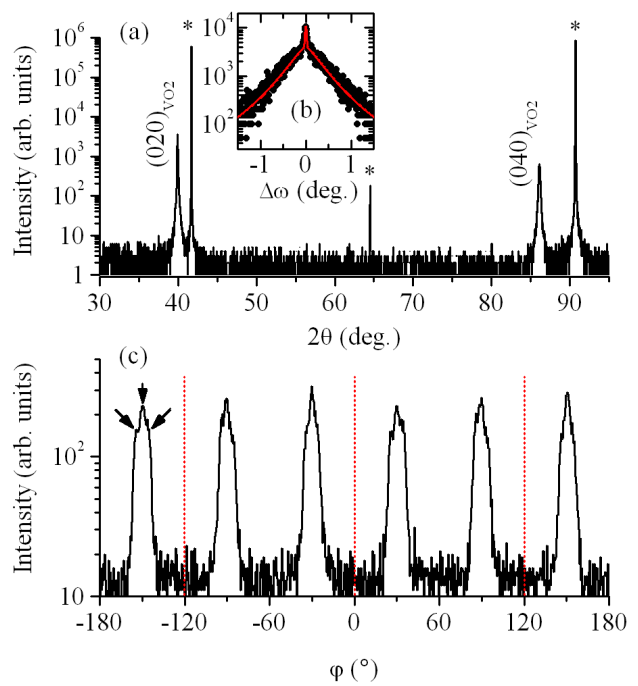

FIG. 1. (a) $\theta-2 \theta$ scan of $100 \mathrm{~nm}$ thick film (zoomed on the $30-95^{\circ}$ region). (b) $\omega$-scan around the (020) reflection of $\mathrm{VO}_{2}$ (dot: data; red line: simulation). (c) $\phi$-scan recorded from the (220) planes of $\mathrm{VO}_{2}$. The dotted lines indicate the positions of the (104) peaks of sapphire.

\section{RESULTS}

\section{A. Film orientation and quality}

Fig. 1(c) displays the $\phi$-scans recorded from the (220) reflection of $\mathrm{VO}_{2}$ in the case of a typical 100 $\mathrm{nm}$ thick film. For the $\mathrm{VO}_{2}$ planes we observe a six-fold symmetry instead of the two-fold symmetry expected for the (220) planes. This discrepancy is well known and is due to the existence of three structurally equivalent orientations of the (010) planes 
with respect to the (001) plane of sapphire, hence giving rise to three in-plane epitaxial variants ${ }^{33,37,38}$ The corresponding epitaxial relationships read: $[100]_{V O 2}\left\|[210]_{A l 2 O 3}, \quad[100]_{V O 2}\right\|[-110]_{A l 2 O 3}$ and $[100]_{V O 2} \|[120]_{A l 2 O 3}$ (in this article we don't make use of the four indices notation for the hexagonal unit-cell). We also notice that the (220) peaks from $\mathrm{VO}_{2}$ are broad and splitted (with satellite peaks appearing at approximately $\pm 2.6^{\circ}$, as indicated by the arrows). This is due to angle mismatch between the $\beta$ angle of $\mathrm{VO}_{2}\left(122.6^{\circ}\right)$ and the $\gamma$ angle of $\mathrm{Al}_{2} \mathrm{O}_{3}\left(120^{\circ}\right)$, as already observed in this system ${ }^{37}$. In the growth mechanism proposed by Chen et al. ${ }^{33}$, the $\mathrm{V}^{4+}$ ions form bonds with the substrate surface $\mathrm{O}^{2-}$ ions and stack on a triangle of the $\mathrm{O}^{2-}$ ions to minimize the electrostatic potential. The interfacial strain hence originates from the distance misfit between $\mathrm{V}^{4+}$ ions in the $\mathrm{VO}_{2}$ structure and the centre of the $\mathrm{O}^{2-}$ triangles in the sapphire structure. This correspond to huge epitaxial strains of $-4.4 \%$ (compressive) along $[100]_{V O 2}$ and $2.1 \%$ (tensile) along $[001]_{V O 2}$.

Such high strains prohibits any possibility of pseudomorphic (lattice-matched) growth. In such cases the growth generally takes place by domain-matching epitaxy where different integral multiples of lattice planes match across the interface, i.e. $n_{f}$ lattice planes of the film match $n_{s}$ lattice planes of the substrate and $n_{f} \neq n_{s}$. The region of bad matching gives rise to a so-called geometric dislocation ${ }^{39,40}$. This growth mechanism has also been observed in (010) $\mathrm{VO}_{2}$ films grown on $\mathrm{NiO}$ buffered substrates $^{15,41}$. This mechanism ensures a strain free growth, so that the only remaining source of elastic strain is due the difference in thermal expansion coefficients between the film and the substrate upon cooling down from the growth temperature, $\mathrm{T}_{g}$. Moreover, as observed experimentally, within this mechanism the lattice distortions are confined close to the geometric dislocations at the interface, ensuring a high crystalline quality despite the high lattice mismatch.

Fig. 1(b) show the $\omega$-scans recorded around the (020) reflection from the same $100 \mathrm{~nm}$ thick film. The peak exhibit a peculiar two-component profile shape, comprising a narrow (coherent) peak superimposed with a broad (diffuse) peak. This behavior is characteristic of thin films in which the random lattice distortions (rotations) are confined into regions of limited spatial extension, quantified by the correlation length $\xi^{42,43}$. The coherent peak then originates from the long range crystalline order (attesting of the film quality), whereas the broad diffuse peak originates from the highly distorted regions. The simulation of such profiles ${ }^{42,44}$ (red curve in Fig. 1(b)) then allows to determine the mosaicity $\varepsilon_{x z}$, the correlation length $\xi$ and the lateral coherence length $D$ (in-plane crystallite size) as long as the coherent peak is visible (this analysis requires the simulation of at least two diffraction orders; for conciseness only the 020 reflection is shown here). From the simulation, it appears that the mosaicity is confined into narrow regions $(\xi=9.5 \mathrm{~nm}$ and a mosaicity of $0.68^{\circ}$ ), in very good agreement with the domain-matching growth mechanism mentioned earlier. The simulation also provides an in-plane crystallite size of $\sim 300-400 \mathrm{~nm}$ for all films considered, without any dependence to the film thickness.

\section{B. Influence of film thickness on the SPT}

The $\theta-2 \theta$ scans recorded around the $(020)$ reflections from $\mathrm{VO}_{2}$ films with increasing thickness have been fitted with pseudo-Voigt functions in order to accurately determine the peak position. The peak position provides, through Bragg's law, the planar spacing $d_{020}$, and consequently the lattice parameter $b=2 d_{020}$ of $\mathrm{VO}_{2}$. The deviation of the observed lattice parameter from its theoretical value $b^{\text {th }}$, will be quantified using the out-of-plane strain:

$$
e_{z z}=\frac{b-b^{t h}}{b^{t h}}
$$

The subscript ' $z z$ ' indicates that this strain correspond to the third diagonal component of the strain tensor, the $z$ axis being chosen normal the surface and the $x$ axis is in the surface of the film in the direction of the X-ray beam. The evolution of the strain for increasing film thickness is given in Fig. 2 . The most striking feature is that the level of strain in the film significantly decreases from a highly strained state $(-0.52 \%)$ to an almost strain-free state $(-0.02 \%)$. The continuous curve is a fit assuming that the strain vs. film thickness obeys a linear behavior, $e_{z z}=a \times t+b$. A moderate scattering (with standard deviation of $0.055 \%$ ) of the strain values around the interpolated curve is observed. This may be explained by the fact that the samples analyzed in this work have been synthesized at different moments in time: the microstructure and the level of strain of the film may be dependent on the wear of the $\mathrm{VO}_{2}$ target used for the film growth. In the following, the results will be discussed as a function of the level of strain.

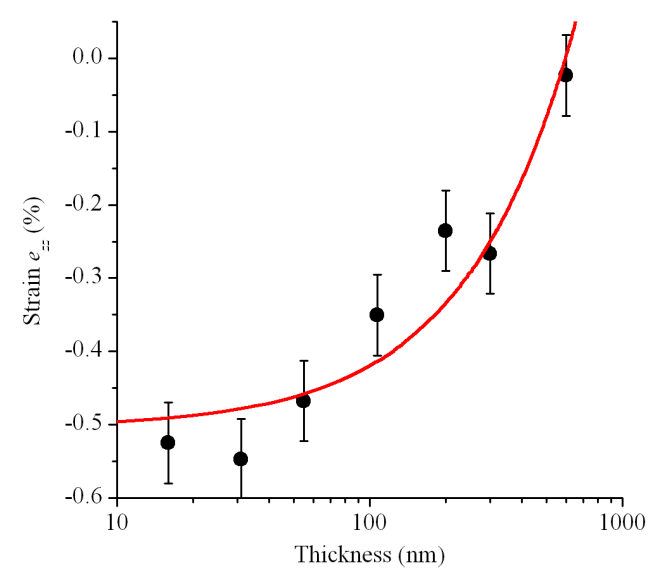

FIG. 2. Evolution of strain with increasing film thickness. The red line is a fit with a linear equation of the type strain $=a \times$ thickness $+b$.

Temperature dependent XRD measurements, per- 
formed in the angular domain containing the 020 and 006 reflections of $\mathrm{VO}_{2}$ and sapphire, respectively, have been recorded for temperatures ranging between 50 and $90^{\circ} \mathrm{C}$ for selected films with different thickness (the substrate peak has been used as an angular reference to account for any possible dilatation-induced sample-displacement errors). The evolution of the (020) reflection for $200 \mathrm{~nm}$ - thick film is displayed in Fig. 3 for selected temperatures across the SPT. Fig. 3(a) and (d) correspond to the $(020)$ reflection from the monoclinic phase, M1 $(2 \theta$ $\left.=39.94^{\circ}\right)$ and the $(200)$ reflection of the tetragonal rutile phase, $\mathrm{R}\left(2 \theta=39.91^{\circ}\right)$ of $\mathrm{VO}_{2}$, respectively. For intermediate temperatures an additional peak, labeled $\mathrm{Mx}$, is clearly observed at $2 \theta=39.89^{\circ}$, revealing the existence of intermediate phase during the transition. In order to extract the volume fraction of each phase during the SPT, the XRD data have been simulated with pseudo-Voigt functions (i.e. a linear combination of a Gaussian and a Lorentzian function). The positions of the M1 and R peaks have been fixed to their respective values as given above, whereas the position of the Mx phase, whose theoretical position is unknown, was allowed to vary during the simulation (the value given above is the result of the simulations and is similar for all investigated samples where the Mx phase is present). The full-width at half maximum (FWHM) and shape parameters $(\eta \in[0,1]$ where 0 corresponds to a Gaussian function and 1 to a Lorentzian function) of the peaks corresponding to the M1 and R phase have been fixed to their respective values observed before and after the transition, respectively. The parameters of the third peak were allowed to vary freely during the simulation procedure. The integrated intensity of each phase can be written ${ }^{45}$ :

$$
I_{i}=C P L m_{h k l}\left|F_{h k l}\right|^{2} V i / \nu_{m, i}
$$

where $V$ is the volume of the phase $i(i=\mathrm{R}, \mathrm{M} 1, \mathrm{Mx})$ irradiated by the x-ray beam, $C$ is a constant depending on the intensity of the incident beam and the counting time, $P$ and $L$ are the polarization and the Lorentz factors, $\nu_{m, i}$ is the unit cell volume of the phase $i$, and $m_{h k l}$ and $F_{h k l}$ are the multiplicity and the structure factor of the considered $h k l$ reflection, respectively. In this special case, because of the epitaxial nature of the film the multiplicity factor is reduced to 1 . Because of the limited temperature range considered in the experiment $\left(25^{\circ} \mathrm{C}\right)$, all temperature-dependent factor other than the phase volume (in particular the unit-cell volume, or the thermal Debye-Waller contribution to the structure factor) can be safely neglected. For instance, whereas the phase volume varies by $100 \%$ across the temperature range considered, the unit-cell volume varies by only $\sim 0.1 \%$, which is smaller than the lowest experimental uncertainty on the measured intensity (which is of the order of $0.2 \%$ ). Therefore the intensity ratio $I_{M 1} / I_{M 1}$ $\left(60^{\circ} \mathrm{C}\right)$ can be considered as a good approximation of the volume fraction $x_{M 1}=V_{M 1} /\left(V_{M 1}+V_{R}+V_{M x}\right)$ of each phase during the transition. Similarly, the volume fraction of the $\mathrm{R}$ phase can be obtained from $x_{R}=I_{R} / I_{R}\left(85^{\circ} \mathrm{C}\right)$. Finally the volume fraction of the intermediate phase follows $x_{x}=1-x_{M 1}-x_{R}$.

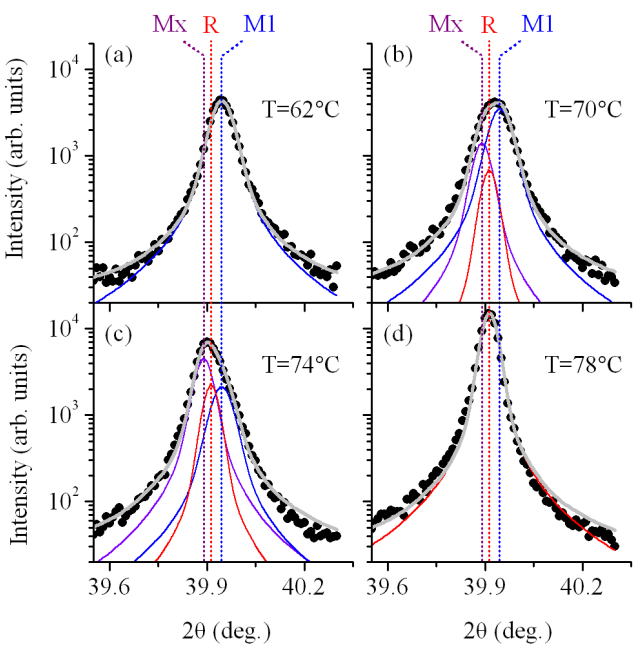

FIG. 3. Evolution of the (020) peak of $\mathrm{VO}_{2}$ during the transition. The peak has been fitted with three pseudo Voigt functions corresponding to the M1 phase (blue line), the R phase (red line) and an intermediate Mx phase (purple line). The grey curve is the sum of all three contributions. The vertical lines indicate the peak positions of the M1, R and Mx phases.
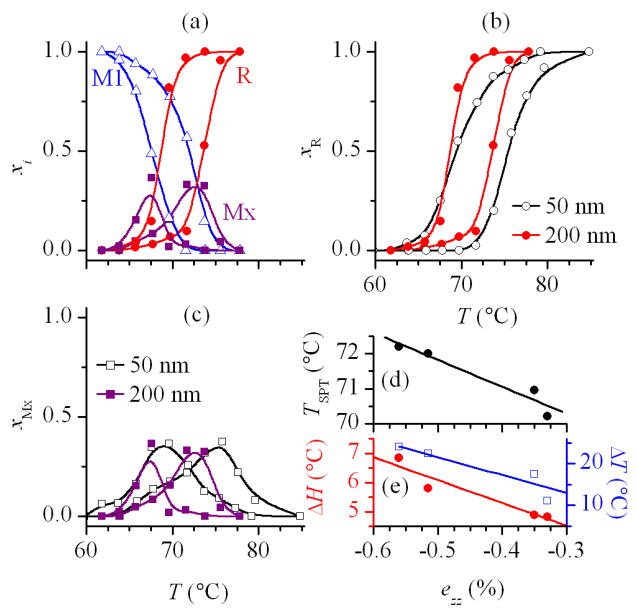

FIG. 4. (a) Temperature evolution of the volume fraction of M1, R and Mx phases as evaluated by XRD for a $200 \mathrm{~nm}$ thick film. (b, c) evolution of the volume fractions of the $\mathrm{R}$ and Mx phases, respectively, for a $50 \mathrm{~nm}$ and $200 \mathrm{~nm}$ - thick films. (d) evolution of the SPT temperature as a function of the out-of-plane strain. The line is an interpolation with a linear function. (e) evolution of the hysteresis width $(\Delta H$, red circles) and temperature range ( $\Delta T$, blue squares). The lines correspond to an interpolation with a linear function.

The evolution of the the volume fractions during the SPT are displayed in Fig. 4(a) for a $200 \mathrm{~nm}$ thick $\mathrm{VO}_{2}$ film. Considering the temperature evolution of the $\mathrm{R}$ phase in Fig. 4(a) and (b), we define $T_{S P T}$ up and 
$T_{S P T}$ down as the transition temperature upon heating and cooling, respectively; $T_{S P T}$ up and $T_{S P T}$ down being determined from the maximum of the derivative $d x_{R} / d T$, upon heating and cooling respectively. The temperature of the SPT is determined from the average $T_{M I T}=\left(T_{S P T}{ }^{\text {up }}+T_{S P T}{ }^{\text {down }}\right) / 2$, whereas the width of the hysteresis curve is defined from the difference $\Delta H=T_{S P T}{ }^{u p}-T_{S P T}$ down. Finally, the smoothness of the transition is defined by the range, $\Delta T$, over which the transition takes place ${ }^{46}$. With these definitions, the $200 \mathrm{~nm}$ thick film exhibits transition temperature $T_{S P T}=70.9^{\circ} \mathrm{C}$ with a $\Delta H=4.8^{\circ} \mathrm{C}$ wide hysteresis loop and spans a $\Delta T=17^{\circ} \mathrm{C}$ temperature range. For a $50 \mathrm{~nm}$ thick film (whose R-phase volume fraction evolution with temperature is compared with the 200-nm thick film in Fig. 4(b)), the hysteresis loop has the same shape, but the SPT is shifted $1^{\circ} \mathrm{C}$ towards higher temperatures, exhibits a wider hysteresis $\left(\Delta H=6^{\circ} \mathrm{C}\right)$ and spans a broader temperature range $\left(\Delta T=22.5^{\circ} \mathrm{C}\right)$. Fig. 4 (c) shows the evolution of the intermediate, $\mathrm{M}_{x}$ phase. For both films (50- and 200-nm thick) the maximum volume fraction is $\sim 0.37$ indicating that this intermediate phase never takes over the whole film volume. However, it is clearly observed that the intermediate phase spans a broader range of temperatures for the $50 \mathrm{~nm}$ thick film $\left(19^{\circ} \mathrm{C}\right.$ instead of $12^{\circ} \mathrm{C}$ for the $200-n m$ thick film), indicating a stabilization of this phase for the lower film thickness. Fig. 4(d,e) summarizes the evolution of the SPT characteristics for all films analyzed by temperaturedependent XRD, as a function of their level of strain. The average transition temperature, Fig. 4(d), the width of the hysteresis (red circles in Fig. 4(e)) and the transition smoothness (blue squares in Fig. 4(e)) are all found to exhibit a monotonic decrease for a decreasing level of strain (i.e. increasing thickness).

\section{Influence of film thickness on the MIT}

Fig. 5(a)-(c) shows the temperature evolution of the electrical resistivity of selected $\mathrm{VO}_{2}$ films with increasing thicknesses. All samples clearly show the metal-insulator transition characteristic of the $\mathrm{VO}_{2}$ material. We observe that the films with a thickness equal or higher than $200 \mathrm{~nm}$ exhibit an abrupt and narrow transition, with a resistivity ratio $\rho\left(25^{\circ} \mathrm{C}\right) / \rho\left(95^{\circ} \mathrm{C}\right)$ ranging over more than 4 orders of magnitude. On the contrary, the thinnest film exhibit degraded electrical properties, a larger width of the hysteresis loop, a transition spanning a broader range of temperature and a lower resistivity ratio $\left(\rho\left(25^{\circ} \mathrm{C}\right) / \rho\left(95^{\circ} \mathrm{C}\right)\right.$ of only 3 orders of magnitude). Similarly to the definitions given above, we have $T_{M I T}=\left(T_{M I T}\right.$ up $+T_{M I T}$ down $) / 2$, $\Delta H=T_{M I T}{ }^{u p}-T_{M I T}{ }^{\text {down }}$, where $T_{M I T}{ }^{u p}$ and $T_{M I T}$ down are defined from the maximum of $d \log (\rho) / d T$ upon heating and cooling, respectively ${ }^{47}$.

In the insulating phase, the electrical conduction can be described by a thermally activated process, so that the resistivity obeys an Arrhenius law: $\rho=\rho_{0} \exp \left(E_{A} / k T\right)$,
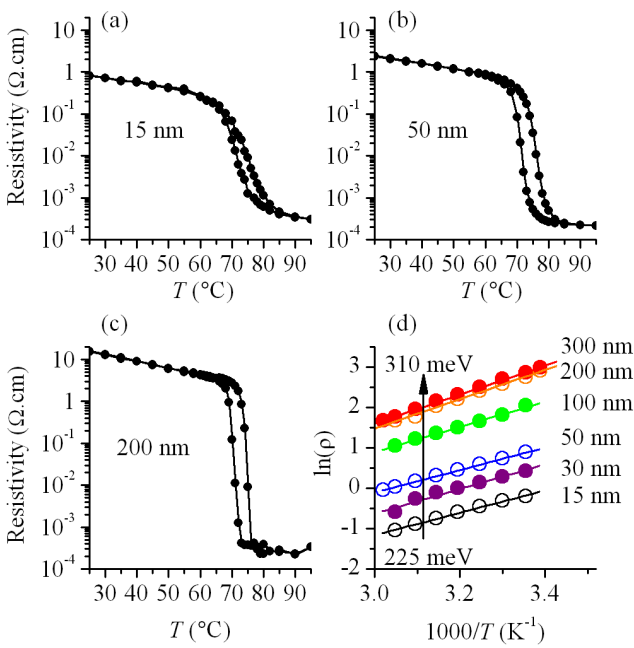

FIG. 5. (a-c) evolution of the resistivity vs. temperature for $\mathrm{VO}_{2}$ films with thickness $15 \mathrm{~nm}, 50 \mathrm{~nm}$ and $200 \mathrm{~nm}$, respectively. (d) Arrhenius plot of the resistivity in the insulating phase for $\mathrm{VO}_{2}$ films with increasing film thickness

where $E_{A}$ is the activation energy, $k$ is boltzmann's constant, and $\rho_{0}$ is the resistivity for $T \rightarrow \infty$. Fig. 5(d) shows a plot of $\ln (r)$ vs. $1 / T$ in the insulating phase, for different film thicknesses, which indeed exhibit a linear behavior. From the slope of the plot we extract the values of $E_{A}$ which range between $0.31 \mathrm{eV}$ for a $300 \mathrm{~nm}$ thick layer and $0.225 \mathrm{eV}$ for the thinnest, $15 \mathrm{~nm}$ thick film. In the case of an intrinsic semi-conductor (i.e. with the Fermi level located in the middle of the band gap), the activation energy is expected to be equal to the half of the optical gap of the $\mathrm{VO}_{2}(0.6 \mathrm{eV})^{48,49}$. The values obtained here, especially for thicknesses higher than $100 \mathrm{~nm}$, are well superior to the ones usually reported in literature for $\mathrm{VO}_{2}$ thin films ${ }^{50,51}$, and are close to the theoretical value of $0.3 \mathrm{eV}$ reported, for instance, in nanoscale single-domain vanadium dioxide nanobeams containing very few defects and impurities ${ }^{52,53}$.

Fig.6 summarizes, for all films, the evolution of the MIT characteristics as a function of the strain extracted from the XRD analysis. The similarity with the characteristics of the SPT is clearly observed: a decrease in the film's level of strain induces a decrease of $\Delta T$, and an increase of the resistivity ratio, indicating an improvement of the electrical properties. Additionally, similarly to what is observed for the SPT, we observe a shift of the MIT temperature towards lower temperature for decreasing strain (or increasing thickness). Contrarily to what is observed for the SPT, though, the width of the hysteresis doesn't seem to be affect by the level of strain or the film thickness. 

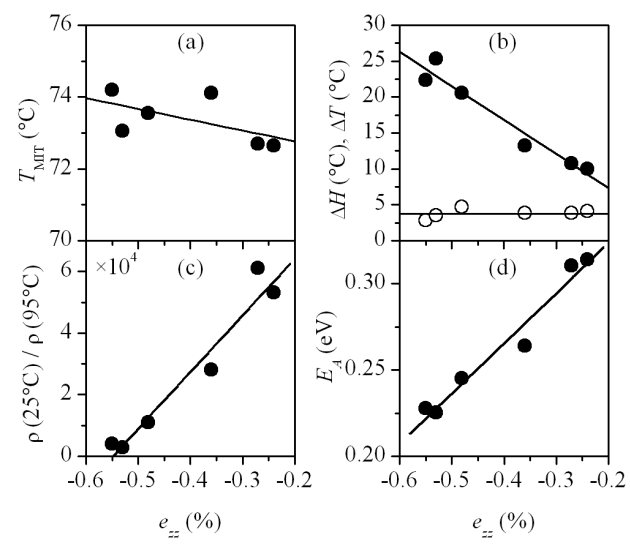

FIG. 6. Evolution of electrical properties with strain: (a) MIT temperature, (b) transition width (filled circles) and hysteresis width (open circles), (c) resistivity ratio and (d) activation energy. The lines are interpolations with linear functions.

\section{DISCUSSION}

\section{A. Origin of the strain}

We shall first discuss the evolution of the homogeneous strain $e_{z z}$. Within the domain-matching epitaxial growth mechanism mentioned earlier, the film grows in a strain relaxed state so that the only possible source for the observed strain is the thermal expansion mismatch between $\mathrm{VO}_{2}$ and the underlying sapphire substrate when cooling down from the growth temperature. The coefficients of thermal expansion of $\mathrm{VO}_{2}$ in the in-plane direction, in the monoclinic (M1) and rutile (R) phases are $\alpha_{b}{ }^{R}=5.83 \times 10^{-6} K^{-1}, \alpha_{c}{ }^{R}=29.7 \times 10^{-6} K^{-1}$ and $\alpha_{a}{ }^{M}=12.1 \times 10^{-6} K^{-1}, \alpha_{c}{ }^{M}=2.57 \times 10^{-6} K^{-1}$, respectively (computed from ${ }^{54,55}$ ), where the subscript a, $\mathrm{b}$ and $\mathrm{c}$ indicate the respective directions of the unit cell. Each of the strain component due the thermal expansion mismatch can be written

$$
\begin{array}{r}
e_{i}^{(t h)}=\left(\alpha_{i}^{(R)}-\alpha_{/ /}^{(A l 2 O 3)}\right)\left(T_{g}-T_{S P T}\right) \\
+\left(\alpha_{i}^{(M)}-\alpha_{/ /}^{(A l 2 O 3)}\right)\left(T_{S P T}-298\right)
\end{array}
$$

where $\alpha_{/ /}{ }^{(A l 203)}=5 \times 10^{-6} K^{-1}$ is the in-plane thermal expansion coefficient of sapphire and, $T_{g}$ is the growth temperature. Computing a simple average thermal expansion coefficient of $\mathrm{VO}_{2}$ in both the $\mathrm{R}$ (from $500^{\circ} \mathrm{C}$ to $68^{\circ} \mathrm{C}$ ) and $\mathrm{M} 1$ (from $68^{\circ} \mathrm{C}$ to $25^{\circ} \mathrm{C}$ ) phases yields a tensile in-plane thermal strain of $0.43 \%$. The out-of-plane elastic strain (Eq. 1) is related to the in-plane strain through $e_{z z}^{(t h)}=-\nu_{2} e_{/ /}$, where $\nu_{2}$ is the biaxial Poisson's ratio, which is itself related to the Poisson's ratio through $\nu_{2}=2 \nu /(1-\nu)$, hence the observed out-of-plane compressive strain.

Computing the corresponding out-of-plane strain requires the knowledge of the Poisson's ratio. For the (100) orientation (corresponding to the (001) planes of the $\mathrm{R}$ phase) the Poisson's ration of $\mathrm{VO}_{2}$ has been recently determined to $0.249^{20}$ which yields $\nu_{2}=0.663$ and therefore a theoretical compressive out-of-plane elastic strain of $e^{(t h)} z z=-0.29 \%$. Since our films are (010) oriented, the corresponding ratio is certainly different. Nonetheless, this value is of the same order of magnitude of the values reported here (Fig. 2).

Let us now focus on the evolution of the strain for varying film thickness. Since the elastic energy scales with the film thickness, strain relaxation is expected to occur for thicker films, whereas the thinnest film should exhibit the highest strain values as given by the thermal strain computed above. The maximum strain is given by the intercept of the interpolating line in Fig. 2, $b$ $=-0.51 \%$. The discrepancy with the value of the thermal strain, $e^{(t h)} z z$, obtained above is very likely due to the fact that we used the Poisson's ratio corresponding to the (100) orientation $\mathrm{VO}_{2}$. Using this result we can compute the Poisson's ratio corresponding to the (010) orientation, and we obtain $\nu=0.372$. The thickest film grown and analyzed in this study $(600 \mathrm{~nm})$ appeared to be heavily cracked $^{56}$, which indicates that the in-plane (tensile) thermal stress has been relieved by the formation of cracks. The residual strain for this film is close to $0(-0.02 \%)$, indicating a complete strain relaxation.

\section{B. Influence of strain on the SPT and the MIT}

Fig. 2 indicates that $\mathrm{VO}_{2}$ films are subjected to inplane tensile strain as a consequence of the film/substrate thermal expansion mismatch, and the thinnest films exhibit a higher level of strain. Figs. 4 and 6 show that a higher in-plane tensile strain yields (i) a shift of the SPT and the MIT towards higher temperatures, (ii) a widening of the temperature range of the SPT and MIT together with a decrease in the resistivity ratio, and finally (iii) a stabilization of the intermediate $\mathrm{Mx}$ phase over a wider range of temperature.

The first observation can be qualitatively understood by examining the dimensional changes of the $\mathrm{VO}_{2}$ unitcell during the SPT. For this purpose, we shall describe both the M1 and the R phase using a common unitcell. The monoclinic equivalent of the $\mathrm{R}$ unit-cell is obtained from $\mathbf{a}_{M}=2 \mathbf{c}_{R}, \mathbf{b}_{M}=\mathbf{a}_{R}$, and $\mathbf{c}_{M}=\mathbf{b}_{R}$ $\mathbf{c}_{R}{ }^{57}$. At the SPT, the $\mathrm{a}_{M}, \mathrm{~b}_{M}$ and $\mathrm{c}_{M}$ lattice parameters vary by $-0.98 \%$ (compressive), $0.60 \%$ (tensile) and $-0.23 \%$ (compressive) ${ }^{54}$. Therefore, the application of tensile strain in the basal $(\mathrm{a}, \mathrm{c})$ plane impedes the SPT and stabilizes the M1 phase, hence the observed shift of the SPT towards higher temperatures under the action of biaxial tensile strain, Fig. 5(d). Interestingly, the extrapolation of $\mathrm{T}_{S P T}$ for a strain free film gives $\mathrm{T}_{S P T}=68^{\circ} \mathrm{C}$ as expected from bulk $\mathrm{VO}_{2}$. The in-plane tensile strain exerted along the $\mathrm{c}_{R}$ axis in our films also explains the shift of the MIT towards higher temperature, in good agreement with previous theoretical and experimental studies ${ }^{15,20,21,27-29,33,34}$. However, in contrast with most theoretical studies ${ }^{17,29}$ where the role of 
strain on the MIT is rationalized in terms of uniaxial strain exerted along the $c_{R}$ axis, which experimentally correspond to the growth on (001) oriented $\mathrm{TiO}_{2}$ substrates, the state of strain is here biaxial: tensile strain is exerted both parallel to $\mathrm{c}_{R}$ and perpendicular to it (parallel to $\mathrm{a}_{R}$ ). This might partly explain why the observed shift of the MIT is here relatively moderate when compared to films grown on (001) $\mathrm{TiO}_{2}$ substrates ${ }^{20,28,29}$.

We also observe that the MIT takes place approximately $2^{\circ} \mathrm{C}$ higher than the SPT. While a decoupling of the MIT and SPT is sometime observed ${ }^{30,31}$, usually the MIT precedes the SPT. In the present case, this temperature difference might be partly explained by the difference in sensitivity of the different techniques used to characterize the MIT and the SPT. The SPT, as characterized by XRD, starts as soon as the first crystallites of the $\mathrm{R}$ (or $\mathrm{Mx}$ ) phase are formed, whereas the MIT requires the $\mathrm{R}$ phase to grow sufficiently to reach the percolation threshold. However, the quantitative analysis of the volume fraction and resistivity data failed to explain the whole temperature shift in terms of percolation phenomena, and a systematic shift of temperature reading between the electrical and the structural characterizations can not be definitely ruled out. Simultaneous electrical and in-situ structural characterizations are required to definitely clarify that point. It must be emphasized, though, that this discrepancy has no consequences on the conclusions drawn in this article since we do not discuss the absolute values of the temperature but only relative evolutions (as above), or temperature differences (as discussed below).

The widening of the SPT and MIT temperature range can be explained by the role played by the film thickness (rather than the level of strain). Indeed, as outlined earlier, the domain-matching epitaxial growth mechanism allows, by the formation of geometric dislocations confined at the interface the growth of high quality films despite huge lattice mismatch values. As a consequence, the regions surrounding the geometric dislocations are in general highly strained. In the case of compounds with cations exhibiting multiple possible oxidation states, it is known than during growth, strain can be partly relieved by promoting local oxidation or reduction of the cations ${ }^{58}$. In the present case, tensile strain would yield $V^{4+}+e \rightarrow V^{3+}$, whereas compressive strain would give $V^{4+} \rightarrow V^{5+}+e$. Since the width of the transition is proportional to the concentration of defects, for the thinner films, where the concentration of defects is increased as a result of the reduced volume of the defect-free region, larger $\Delta T$ values are observed ${ }^{15}$, for both the MIT and the SPT.

This scenario is confirmed by the evolution of the activation energy, $E_{A}$, with the film thickness. With increasing strain (i.e. decreasing film thickness), Fig. 6(d), the defective interface region has a higher influence on the their electrical properties. Most likely, the presence of the above-mentioned crystalline defects introduce (donor or acceptor) defect levels in the band gap of the films ${ }^{12,14}$ which directly influence the resistivity in the insulating phase of the films and might explain the observed decrease of the activation energy for the lower film thickness. In contrast, as suggested above, the thickest films (> $100 \mathrm{~nm}$ ) have a reduced proportion of interface-induced defects and exhibit activation energies close to the expected value for stoichiometric, high quality $\mathrm{VO}_{2}$ crystals ${ }^{52,53}$.

The width of the hysteresis of the MIT is believed to be dependent on the crystallite $\operatorname{size}^{15}$. Our XRD experiments reveal that the crystallite size is independent on the film thickness which can explain why the $\Delta H$ does not vary in the case of the MIT. On the other hand, the increase of $\Delta H$ for the SPT can be viewed as a consequence of the stabilization of the $\mathrm{Mx}$ phase. Whereas strain free undergo a simple $\mathrm{M} 1 \rightarrow \mathrm{R}$ transition, strained films exhibit the following sequence $\mathrm{M} 1 \rightarrow \mathrm{M} 1$ $+\mathrm{Mx} \rightarrow \mathrm{Mx}+\mathrm{R} \rightarrow \mathrm{R}$ (see Fig. 3), hence the broader hysteresis.

The last observation, regarding the intermediate phase, is less easily explained. There have been numerous reports of intermediate phases occurring during the SPT from the M1 to the $\mathrm{R}$ phase, the most common being the M2 phase ${ }^{16,19,26,57,59,60}$, but other phase were also reported: $\mathrm{M}^{57}, \mathrm{~T}^{19,61}, \mathrm{~A}^{62,63}, \mathrm{~B}^{62,64}, \mathrm{R}$-like ${ }^{65}$ and other disordered or unidentified phases ${ }^{30,31,66}$. Assessing the exact nature of this intermediate phase is a challenging task. In a similar situation of $\mathrm{VO}_{2}$ films grown on (001) sapphire by pulsed laser deposition and reactive sputtering Okimura et al. ${ }^{60}$ reported the existence of the M2 phase. The M2 phases is known to be stabilized under the action of tensile strain along the $\mathrm{c}$ direction of the $\mathrm{R}$ phase ${ }^{26}$. The stabilization of the intermediate phase for the lowest film thickness observed can hence be rationalized by its increased level of strain. However, in contrast with Ref. ${ }^{60}$ the intermediate phase observed here is only visible in the $60-85^{\circ} \mathrm{C}$ range and totally vanishes above $85^{\circ} \mathrm{C}$, whereas Okimura et al. reported the M2 phase to appear close to room temperature and, surprisingly, it persisted above the SPT. Additionally, contrarily with our results, they observed the XRD peak of the M2 phase to be located between the (020) and (200) peaks of the M1 and R phase and attributed it to the (002) reflection of the M2 phase ${ }^{60}$. Our results indicate that the intermediate phase has a lattice parameter larger than both the M1 and $\mathrm{R}$ phase. These results cast some doubt regarding the exact nature of the intermediate phase, but a similar transient phase with enlarged unit-cell have been observed in other studies as well ${ }^{66}$. The same behavior has been observed in $\mathrm{VO}_{2}$ nanowires grown on sapphire and the additional phase was interpreted as a strained $\mathrm{R}$ phase ${ }^{67}$, although it is not possible, with the present data, to confirm that the phase observed here exactly correspond to those observed in ${ }^{66}$ or $^{67}$.

Fig. 7 shows the evolution of Raman spectra with increasing temperature for a $100-\mathrm{nm}$ thick annealed $\mathrm{VO}_{2}$ layer. At room temperature, the spectrum is indica- 


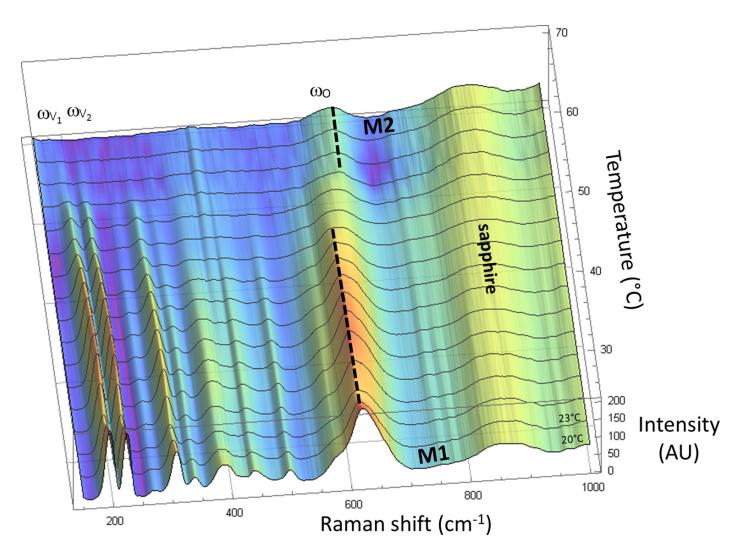

FIG. 7. Raman spectra of a $\mathrm{VO}_{2}$ film (100-nm thick) showing the temperature evolution of the characteristic phonon modes.

tive of the monoclinic $\mathrm{M} 1 \mathrm{VO}_{2}$ phase and is consistent with previous reports on $\mathrm{VO}_{2}$ micro-crystalline thin films and single-crystal microbeams ${ }^{61,68,69}$. The M1 phase is identified mainly by the dominant $\omega_{0}$ phonon frequency at $\sim 615 \mathrm{~cm}^{-1}$ (V-O mode), and the $\omega_{v 1}$ and $\omega_{v 2}$ modes at $195 \mathrm{~cm}^{-1}$ and $225 \mathrm{~cm}^{-1}$, respectively, associated with the $\mathrm{V}-\mathrm{V}$ lattice motion ${ }^{61,68}$. With increasing temperature, in the interval $45-75^{\circ} \mathrm{C}$, the $\omega_{0}$ phonon peak is getting larger, less intense and is slightly redshifted. For higher temperatures the Raman spectra become featureless, characteristic of the metal-like $\mathrm{VO}_{2}$. The frequency shift of the Raman modes with increasing temperature was explained by the onset of a M2 strain-mediated phases in the film, prior to its transition to the metallic $\mathrm{R}$ phase ${ }^{70}$. However, for crystalline $\mathrm{VO}_{2}$ micro-beams submitted to tensile or compressive stress, the M1-R phase transition seems to be far more complex: their strain-temperature phase diagram suggest complex M1-R path transitions involving either the insulating triclinic T phase, the M2 phase, or both of them ${ }^{61}$ . Compared to the Raman signals from single-crystalline $\mathrm{VO}_{2}$ material, the temperature-related Raman peaks of the $\mathrm{VO}_{2}$ analyzed thin film are broader, making difficult to asses/ discern the exact contributions from additional T or M2 phases. Nevertheless, qualitatively, the temperature-evolution of the Raman spectra in our $\mathrm{VO}_{2}$ thin film suggests that the M1-R phase transition seems to evolve through an intermediate, stress-related phase, although further work is required to clearly identify the exact nature of this intermediate phase.

\section{CONCLUSIONS}

High quality (010)-oriented $\mathrm{VO}_{2}$ epitaxial films have been grown on (001) sapphire substrates by means of electron-beam evaporation. Their electrical and structural properties have been characterized by four-probe resistivity measurements on one hand, and Raman spectroscopy and temperature-dependent XRD experiments, on the other hand. The highly-mismatched $\mathrm{VO}_{2} / \mathrm{Al}_{2} \mathrm{O}_{3}$ interface promotes a domain-matching epitaxial growth process where the films grow in a strain-relaxed state and the lattice distortions are confined at the interface in regions with limited spatial extent $(\xi=9.5 \mathrm{~nm})$. Upon cooling down from the growth temperature, tensile strain is stored in the films as a consequence of the thermal expansion mismatch between $\mathrm{VO}_{2}$ and $\mathrm{Al}_{2} \mathrm{O}_{3}$. We examined the role of the magnitude of the strain on both the MIT and the SPT. The thinnest film exhibit the highest level of tensile strain in the $(\mathrm{a}, \mathrm{c})$ of $\mathrm{VO}_{2}$, resulting in a shift of both the MIT and the SPT towards higher temperature pointing to a stabilization of the monoclinic and insulating phase. Concomitantly, the switching characteristics are altered (lower resistivity ratio and broader transition) as result of the presence of structural defects located at the interface. The SPT exhibits a similar evolution with, additionally, a broader hysteresis due to the formation of an intermediate phase in the M1-R transition. Conversely, the thickest films $(600 \mathrm{~nm})$ are fully strain-relaxed and heavily cracked making them unsuitable for device applications. Films with a thickness ranging between 100 and 300 nm undergo a partial strain relaxation and exhibit improved switching characteristics, with a sharp $\left(10^{\circ} \mathrm{C}\right.$ temperature range) and narrow (hysteresis $<4^{\circ} \mathrm{C}$ ) MIT extending over more than four orders of magnitude in resistivity $\left(6 \times 10^{4}\right)$.

\section{ACKNOWLEDGMENTS}

VT is grateful to région Limousin (France) and Labex SigmaLim for financial support.
${ }^{1}$ F. Morin, Phys. Rev. Lett. 3, 34 (1959).

2 A. Cavalleri, C. Tóth, C. W. Siders, J. A. Squier, F. Ráksi, P. Forget, and J. C. Kieffer, Phys. Rev. Lett. 87, 237401 (2001).

3 G. Stefanovich, A. Pergament, and D. Stefanovich, J. Phys. Condens. Matter 12, 8837 (2000).

${ }^{4}$ D. Ruzmetov, G. Gopalakrishnan, C. Ko, V. Narayanamurti, and S. Ramanathan, J. Appl. Phys. 107, 114516 (2010).
5 J. Leroy, A. Bessaudou, F. Cosset, and A. Crunteanu, Thin Solid Films 520, 4823 (2012).

6 Z. Yang, C. Ko, and S. Ramanathan, Annu. Rev. Mater. Sci. 41, 337 (2011), http://dx.doi.org/10.1146/annurevmatsci-062910-100347.

7 F. Dumas-Bouchiat, C. Champeaux, A. Catherinot, A. Crunteanu, and P. Blondy, Appl. Phys. Lett. 91, 223505 (2007).

8 S. D. Ha, Y. Zhou, C. J. Fisher, S. Ramanathan, and J. P. Treadway, J. Appl. Phys. 113, 184501 (2013). 
9 S. Lysenko, A. Rúa, V. Vikhnin, F. Fernández, and H. Liu, Phys. Rev. B 76, 035104 (2007).

${ }^{10}$ M. Rini, Z. Hao, R. W. Schoenlein, C. Giannetti, F. Parmigiani, S. Fourmaux, J. C. Kieffer, A. Fujimori, M. Onoda, S. Wall, and A. Cavalleri, Appl. Phys. Lett. 92, 181904 (2008).

11 H.-T. Kim, B.-G. Chae, D.-H. Youn, S.-L. Maeng, G. Kim, K.-Y. Kang, and Y.-S. Lim, New J. Phys. 6, 52 (2004).

12 Y. Chang, J. Yang, Y. Kim, D. Kim, and T. Noh, Phys. Rev. B 76, 075118 (2007).

13 T.-H. Yang, C. Jin, H. Zhou, R. Narayan, and J. Narayan, Appl. Phys. Lett. 97, 072101 (2010).

14 C. Chen, Y. Zhao, X. Pan, V. Kuryatkov, A. Bernussi, M. Holtz, and Z. Fan, J. Appl. Phys. 110, 023707 (2011).

15 R. Molaei, R. Bayati, F. Wu, and J. Narayan, J. Appl. Phys. 115, 164311 (2014).

16 V. Eyert, Annals of Physics 11, 9 (2002).

17 B. Lazavorits, K. Kim, K. Haule, and G. Kotliar, Phys. Rev. B 81, 115117 (2010).

18 Y. Gu, J. Cao, J. Wu, and L.-Q. Chan, J. Appl. Phys. 108, 083517 (2010).

19 J. Pouget, H. Launois, J. D'Haenens, P. Merenda, and T. Rice, Phys. Rev. Lett. 35, 873 (1975).

20 N. Aetukuri, A. Gray, M. Drouard, M. Cossale, L. Gao, A. Reid, R. Kukreja, H. Ohlag, C. Jenkins, E. Arenholz, K. Roche, H. Dürr, M. Samant, and S. Parkin, Nat. Phys. 10, 1038 (2013).

${ }^{21}$ E. Abreu, M. Liu, J. Lu, K. G. West, S. Kittiwatanakul, W. Yin, S. A. Wolf, and R. D. Averitt, New J. Phys. 14, 083026 (2012).

22 J. Wu, Q. Gu, B. Guiton, N. de Leon, L. Ouyang, and H. Park, Nano Lett. 6, 2313 (2006).

23 B. Hu, Y. Ding, W. Chen, D. Kulkarni, Y. Shen, V. Tsukruk, and Z. Wang, Adv. Mater. 22, 5134 (2010).

${ }^{24}$ L. Bai, Q. Li, S. A. Corr, Y. Meng, C. Park, S. V. Sinogeikin, C. Ko, J. Wu, and G. Shen, Phys. Rev. B 91, 104110 (2015).

${ }^{25}$ H. Asayesh-Ardakani, A. Nie, P. Marley, Y. Zhu, P. Phillips, S. Singh, F. Mashayek, G. Sambandamurthy, K.-b. Law, R. Klie, S. Baerjee, G. Odegard, and R. Shahbazian-Yassar, Nano Lett. 15, 7179 (2015).

26 J. Cao, Y. Gu, L. Chan, D. Ogletree, K. Chan, N. Tamura, M. Kunz, C. Barrett, J. Seidel, and J. Wu, Nano Lett. 10, 2667 (2010).

27 Y. Muraoka and Z. Hiroi, Appl. Phys. Lett. 80, 583 (2002).

${ }^{28}$ H. Paik, J. A. Moyer, T. Spila, J. W. Tashman, J. A. Mundy, E. Freeman, N. Shukla, J. M. Lapano, R. EngelHerbert, W. Zander, J. Schubert, D. A. Muller, S. Datta, P. Schiffer, and D. G. Schlom, Appl. Phys. Lett. 107, 163101 (2015).

29 L. Fan, S. Chan, Z. Luo, Q. Liu, Y. Wu, L. Song, D. Ji, P. Wang, W. Chu, C. Gao, C. Zou, and Z. Wu, Nano Lett. 14, 4036 (2014).

30 B. Kim, Y. Lee, S. Choi, J.-W. Lim, S. Yun, and H.-T. Kim, Phys. Rev. B 77, 235401 (2008).

31 Z. Tao, T.-R. T. Han, S. D. Mahanti, P. M. Duxbury, F. Yuan, C.-Y. Ruan, K. Wang, and J. Wu, Phys. Rev. Lett. 109, 166406 (2012).

${ }^{32}$ G. Xu, P. Jin, M. Tazawa, and K. Yoshimura, Appl. Surf. Sci. 244, 449 (2005).

33 C. Chen, Y. Zhu, Y. Zhao, J. Lee, H. Wang, et al., Appl. Phys. Lett. 97, 211905 (2010).

${ }^{34}$ K. Okimura and J. Sakai, Jpn. J. Appl. Phys. 48, 045504 (2009).

35 X. Li, A. Gloter, H. Gu, X. Cao, P. Jin, and C. Colliex, Acta Mater. 61, 6443 (2013).
36 J. Leroy, A. Crunteanu, A. Bessaudou, F. Cosset, C. Champeaux, and J.-C. Orlianges, Appl. Phys. Lett. 100, 213507 (2012).

37 L. Fan, Y. Wu, C. Si, G. Pan, C. Zou, and Z. Wu, Appl. Phys. Lett. 102, 011604 (2013).

38 F. Wong, Y. Zhou, and S. Ramanathan, J. Cryst. Growth 364, 74 (2013).

39 A. Trampert and K. Ploog, Cryst. Res. Technol. 35, 793 (2000).

40 J. Narayan and B. C. Larson, J. Appl. Phys. 93, 278 (2003).

41 A. Gupta, J. Narayan, and T. Dutta, Appl. Phys. Lett. 97, 151912 (2010).

42 A. Boulle, R. Guinebretière, and A. Dauger, J. Phys. D: Appl. Phys. 38, 3907 (2005).

43 A. Boulle, R. Guinebretière, and A. Dauger, J. Appl. Phys. 97, 073503 (2005).

44 A. Boulle, S. Kilburger, P. D. Bin, E. Millon, C. D. Bin, R. Guinebretière, and A. Bessaudou, J. Phys. D: Appl. Phys. 42, 145403 (2009).

45 B. Warren, X-ray Diffraction, edited by A. wesley:New York (Addison-wesley:New-York, 1969).

46 The beginning of the transition is defined, upon heating, by the temperature at which another phase is observed in the XRD patterns, in addition to the M1 phase. Conversely, the end of the transition is defined when only the $\mathrm{R}$ phase is observed in the XRD patterns. The same procedure is used for cooling.

47 In the case of the $\Delta T$ for the MIT, the beginning (end) of the transition is defined as the temperature at which the resistivity deviates by more than $10 \%$ of its initial slope upon heating (cooling).

48 C. N. Berglund and H. J. Guggenheim, Phys. Rev. 185, 1022 (1969).

49 H. W. Verleur, A. S. Barker, and C. N. Berglund, Phys. Rev. 172, 788 (1968).

50 D. Fu, K. Liu, T. Tao, K. Lo, C. Cheng, B. Liu, R. Zhang, H. A. Bechtel, and J. Wu, J. Appl. Phys. 113, 043707 (2013).

51 J.-C. Orlianges, J. Leroy, A. Crunteanu, R. Mayet, P. Carles, and C. Champeaux, Appl. Phys. Lett. 101, 133102 (2012).

52 J. Cao, W. Fan, H. Zheng, and J. Wu, Nano Lett. 9, 4001 (2009), http://dx.doi.org/10.1021/nl902167b.

53 J. Wei, Z. Wang, W. Chen, and C. D. H., Nature Nanotech. 4, 420 (2009).

54 D. Kucharczyk and T. Niklewski, J. Appl. Crystallogr. 12, 370 (1979).

55 K. V. K. Rao, S. V. N. Naidu, and L. Iyengar, J. Phys. Soc. Jpn. 23, 1380 (1967), http://dx.doi.org/10.1143/JPSJ.23.1380.

56 The film cracking prevented any reliable electrical measurement to be performed. Additionally, even a moderate heating leads the film to flake off thus, preventing any temperature-dependent measurement.

57 M. Marezio, B. McWhan, J. Remeika, and P. Dernier, Phys. Rev. B 5, 2541 (1972).

${ }^{58}$ F. Conchon, A. Boulle, R. Guinebretière, C. Girardot, S. Pignard, J. Kreisel, F. Weiss, E. Dooryhée, and J.-L. Hodeau, Appl. Phys. Lett. 91, 192110 (2007).

59 J. Park, J. Coy, T. Kasirga, C. Huang, Z. Fei, S. Hunter, and D. Cobden, Nature 500, 431 (2013).

${ }^{60}$ K. Okimura, J. Sakai, and S. Rmanathan, J. Appl. Phys. 107, 063503 (2010).

61 J. M. Atkin, S. Berweger, E. K. Chavez, M. B. Raschke, J. Cao, W. Fan, and J. Wu, Phys. Rev. B 85, 020101 
(2012)

62 C. Leroux, G. Nihoul, and G. Van Tendeloo, Phys. Rev. B 57, 5111 (1998).

63 S. R. Popuri, A. Artemenko, C. Labrugere, M. Miclau, A. Villesuzanne, and M. Pollet, J. Solid State Chem. 213, 79 (2014).

64 A. Chen, Z. Bi, W. Zhang, J. Jian, Q. Jia, and H. Wang, Appl. Phys. Lett. 104, 071909 (2014).

${ }^{65}$ H. Qiu, M. Yang, Y. Dong, H. Xu, B. Hong, Y. Gu, Y. Yang, C. Zou, Z. Luo, and C. Gao, New J. Phys. 17, 113016 (2015).

${ }^{66}$ H. Wen, L. Guo, E. Barnes, J. Lee, D. Walko, R. Schaller, J. Moyer, R. Misra, Y. Li, E. Dufresne, D. Schlom,
V. Gopalan, and J. Freeland, Phys. Rev. B 88, 165424 (2013).

67 J. I. Sohn, H. J. Joo, D. Ahn, H. H. Lee, A. E. Porter, K. Kim, D. J. Kang, and M. E. Welland, Nano Lett. 9, 3392 (2009), http://dx.doi.org/10.1021/nl900841k.

${ }^{68}$ J. I. Sohn, H. J. Joo, K. S. Kim, H. W. Yang, A.-R. Jang, D. Ahn, H. H. Lee, S. Cha, D. J. Kang, J. M. Kim, and M. E. Welland, Nanotechnology 23, 205707 (2012).

69 K. Okimura, N. Hanis Azhan, T. Hajiri, S.-i. Kimura, M. Zaghrioui, and J. Sakai, J. Appl. Phys. 115, 153501 (2014).

70 M. Nazari, Y. Zhao, V. V. Kuryatkov, Z. Y. Fan, A. A. Bernussi, and M. Holtz, Phys. Rev. B 87, 035142 (2013). 